\title{
Quasielectrostatic Wave Propagation Beyond the Delay-Bandwidth Limit in Switched Networks
}

\author{
Mykhailo Tymchenko, ${ }^{1}$ Dimitrios Sounas, ${ }^{1,2}$ Aravind Nagulu, ${ }^{3}$ Harish Krishnaswamy, ${ }^{3}$ and Andrea Alù ${ }^{1,4,5,6, *}$ \\ ${ }^{1}$ Department of Electrical and Computer Engineering, The University of Texas at Austin, \\ 1 University Station C0803, Austin, Texas 78712, USA \\ ${ }^{2}$ Department of Electrical and Computer Engineering, Wayne State University, \\ Detroit, Michigan 48202, USA \\ ${ }^{3}$ Department of Electrical Engineering, Columbia University, 1300 South West Mudd, \\ 500 West 120th Street, New York, New York 10027, USA \\ ${ }^{4}$ Photonics Initiative, Advanced Science Research Center, City University of New York, \\ New York, New York 10031, USA \\ ${ }^{5}$ Physics Program, Graduate Center, City University of New York, New York, New York 10026, USA \\ ${ }^{6}$ Department of Electrical Engineering, City College of New York, New York, New York 10031, USA
}

(Received 15 October 2018; revised manuscript received 10 May 2019; published 31 July 2019)

\begin{abstract}
The delay-bandwidth limit implies a stringent trade-off between the time delay, bandwidth, and propagation distance of an electromagnetic signal. Here, we show that temporal modulation can overcome this constraint, enabling extremely broadband wave propagation with close-to-zero group velocity dispersion in switched multipath electronic networks. Contrary to time-invariant waveguides, in which wave propagation implies a delicate balance between electric and magnetic stored energies, in such modulated networks the stored energy is largely electrostatic in nature. We show that in this case the phase and group velocities become independent of the properties of their constituent elements, and they are controlled only by the modulation scheme. Based on these findings, we provide practical designs of deeply subwavelength CMOS-compatible reciprocal and nonreciprocal microwave components, such as delay lines, phase shifters, couplers, and circulators. The obtained results also explicitly show that temporally modulated systems are not bound by constraints of time-invariant systems and can achieve arbitrarily large delay-bandwidth products.
\end{abstract}

DOI: 10.1103/PhysRevX.9.031015

\section{INTRODUCTION}

Spatial modulation and periodicity offer exciting opportunities for modern engineering, allowing one to rely less on the intrinsic properties of natural media and, instead, obtain synthetic collective responses by combining several materials with distinct properties in judicially engineered structures. In electromagnetics, the use of spatial modulation enables a large degree of control over the phase and group velocities, polarization, and amplitude of traveling waves. Examples of such manipulation include extreme light traveling regimes, such as propagation with ultralow $\left(v_{g} \ll c\right)$ and superluminal $\left(v_{g}>c\right)$ group velocities [1-5]. Based on these principles, it has been shown that it is possible to bring a light pulse to a near standstill [6-8].

\footnotetext{
*To whom correspondence should be addressed. aalu@gc.cuny.edu

Published by the American Physical Society under the terms of the Creative Commons Attribution 4.0 International license. Further distribution of this work must maintain attribution to the author(s) and the published article's title, journal citation, and DOI.
}

Subject Areas: Metamaterials, Photonics

Such dramatic modifications of group velocity are typically accompanied by strongly enhanced light-matter interactions which are critically important for electronics, nanophotonics, optical communications, quantum information processing, light harvesting, and sensing. At the same time, many of these applications require that light pulses are not only trapped for prolonged times, but also released without amplitude and phase distortions. This requirement is a key difference between structures aimed at light trapping and those operating as photonic buffers. For instance, light trapping can be obtained by leveraging nonresonant and thus extremely wideband wedge states at the end of adiabatically tapered reciprocal or nonreciprocal waveguides $[9,10]$. The lifetime and bandwidth of such trapped states are determined only by their aggregated outcoupling, radiative and intrinsic loss rate [11]. In turn, to obtain pulse delays significantly larger than those provided by the waveguide dispersion, time-invariant slow-light structures employ resonant cavities which introduce additional group delays in the vicinity of their resonance frequencies. Arranging such cavities in periodic and quasiperiodic lattices enables increased bandwidths and provides better control over the guided mode dispersion. In particular, adiabatically varying ("chirped") structures can be used to 
flatten the dispersion over a certain range of frequencies and obtain distortion-free pulse delays [2]. Still, due to their intrinsically resonant nature, all time-invariant slow-wave structures face a stringent trade-off between the pulse delay $\Delta t=L / v_{g}$, where $L$ is the propagation length and $v_{g}$ is the group velocity, and the available bandwidth $\Delta f$ : For a fixed footprint, the larger the group delay, the smaller the bandwidth. This trade-off can be easily understood if we consider a waveguide at its cutoff: The group velocity approaches zero, leading to an infinitely large delay $\Delta t$, but this process happens essentially at a single frequency, i.e., $\Delta f \rightarrow 0$. It can be also shown that the group velocity achievable in a timeinvariant system is directly proportional to its bandwidth, $v_{g} \propto \Delta f$ [12]. Thus, the delay-bandwidth product (DBP) $\Delta t \cdot \Delta f$ of any time-invariant system is always finite, since it inherently determines how many pulses (or bits) the system can store. The DBP is commonly used as a figure of merit to benchmark and compare the performance of slow-wave structures [13].

All these considerations also apply to time-invariant electronic systems and circuits, except that lumped $L C$ tanks or micromechanical resonators are typically used instead of optical resonators. Wideband electronic delay elements, often called true time delays (TTDs), which are the primary subject of this work, are critical components in the design of analog finite impulse response filters, which find widespread application in equalizers [14], wideband beam formers [15], and interference cancellers [16]. However, in electronic systems the physical size and low quality factor of inductors impose additional constraints on practically attainable group delays. Moreover, since the inductance of a resonant $L C$ circuit grows proportionally to the inverse of the resonance frequency squared, the problem of realizing TTDs is dramatically amplified at frequencies below a few gigahertz.

Breaking time invariance is a powerful method to gain additional control over wave propagation. In time-modulated optical systems, slow- and fast-light regimes can be realized by inducing coherent population oscillations in absorbing media [5], via stimulated Brillouin scattering from a traveling acoustic wave [5,17-19], as well as Raman scattering $[20,21]$, in order to obtain a narrow frequency window with strong refractive index dispersion. Even though these systems suffer from bandwidth limitations, they possess a valuable advantage of being dynamically controllable and reconfigurable. Another interesting method to obtain a slowwave regime and achieve optical buffering is by adiabatically tuning certain properties of the system, such as the resonance frequency of an array of optical cavities or their coupling to bus waveguides, as the pulse enters and exits the system. This approach enables adiabatic compression of the spectral width of the incoming pulse and its coherent storage in an array of high- $Q$ resonators, with subsequent adiabatic decompression and release [12,22]. A more traditional technique is to trap the pulse in a looped waveguide resonator having a round trip which is much larger than the pulse duration and allow the pulse to exit after an integer number of round trips [23-28]. In this case, the evolution of the system is nonadiabatic, since it occurs on timescales much shorter than the periodicity of the field in the resonator. Similarly, trapping of a narrow-band optical pulse in a photonic crystal nanocavity has also been demonstrated [29]. Importantly, the pulse storage time in such systems is limited only by the lifetime of photons in optical cavities, and thus they are not bound by the DBP limit of time-invariant systems. The downside of both approaches, adiabatic and nonadiabatic, is the fact that the catch-and-release process must be performed synchronously with the incoming pulse, which in practice requires a part of the pulse energy to be used for control and synchronization of the tuning network. In addition, dynamic catch-and-release imposes a constraint on spacing between subsequent pulses, which cannot be made smaller than the pulse storage time, implying another limitation on the system bandwidth. Nevertheless, these works clearly show that temporal modulation provides a practical way to overcome the DBP limit of time-invariant systems [30].

Inspired by all these interesting developments, in this paper, we show that synchronized spatial and temporal periodic modulation may be used to fundamentally overcome the delay-bandwidth limit of time-invariant systems by breaking the dependence of phase and group velocities on the properties of the constituent elements. Over the past few years, spatiotemporal modulation has gained significant attention, in particular, due to the possibility of realizing miniaturized nonreciprocal components enabling the realization of magnet-free isolators [31-34], phase shifters [35], miniaturized radio frequency gyrators, and circulators [36-44] - important breakthroughs on the way towards integrated communication systems. However, all these nonreciprocal devices, as well as many reciprocal ones, rely on large delays that provide the necessary phase shifts among their constituent subunits, fundamentally limiting their further miniaturization. In the following, we use a specific form of nonadiabatic spatiotemporal modulationswitching - in multipath electronic networks to realize deeply subwavelength reciprocal and nonreciprocal delay lines exhibiting near-zero group velocity dispersion over extremely broad bandwidths. We also show that, instead of being functions of the impedance of the involved circuit elements, the phase and group velocities in these networks are inherently defined by the modulation scheme, enabling dynamic delay-bandwidth reconfiguration, well beyond the limits of static schemes. These highly unusual properties stem from the fact that, while conventional delay lines and resonators are bound to store nearly equal amounts of electric and magnetic energy, the proposed switched networks store energy mostly electrostatically, opening a new regime of electromagnetic wave propagation. Building upon these findings, we present realistic designs of miniaturized 
reciprocal and nonreciprocal devices that can successfully replace arbitrarily long delay line sections within extremely compact footprints, as well as synthesize various phasenonreciprocal responses. This opens exciting prospects for the realization of deeply subwavelength and fully integrable reciprocal and nonreciprocal electronic devices and systems based on spatiotemporal modulation.

\section{COMMUTATED NETWORKS AS CAPACITIVE PHASE SHIFTERS}

Consider a network consisting of a periodic ladder of identical linear time-invariant (LTI) banks, each of which is connected to the input and output ports through frequency mixers or modulators, for example, switches [45,46]. Figure 1(a) shows an example of such a two-port network consisting of $N$ branches, each containing a shunt capacitance $C$ connected to the ports through two switches. We assume the input and output port impedances to be $Z_{0}$. The two sets of switches are commutated in a staggered fashion, periodically connecting and disconnecting each capacitor. On either side of the network, only one switch is closed at any moment in time, and it remains closed over a time interval $T_{s} / N$, where $T_{s}$ is the switching period. Additionally, due to the staggering, the two switches on the same path never close at the same time; i.e., the output set of switches lags the input set by a time delay $\Delta T=[1,2, \ldots, N-1] \times T_{s} / N$, corresponding to a commutation phase shift $\gamma=-2 \pi \Delta T / T_{s}$.

The properties of the network at each frequency are defined by the charge or discharge time constant $\tau=Z_{0} C$, switching frequency $f_{s}=1 / T_{s}$, and the number of paths $N$. Depending on these parameters, the network can exhibit remarkably different behaviors. When the time interval over which each switch is closed is much smaller than the time constant of the shunt capacitor $\tau \gg T_{s} / N$, such a network synthesizes the response of a high- $Q$ comb filter commonly referred to as an $N$-path filter which exhibits a series of narrow transmission peaks centered at $f=m f_{s}$, where $m=0,1,2, \ldots[45,46,48]$. Conventional time-invariant bandpass filters require the use of inductors, leading to large footprints, difficult integration, poor efficiency, and low $Q$ factors, especially at low frequencies. In turn, the width of transmission peaks of $N$-path filters (a)

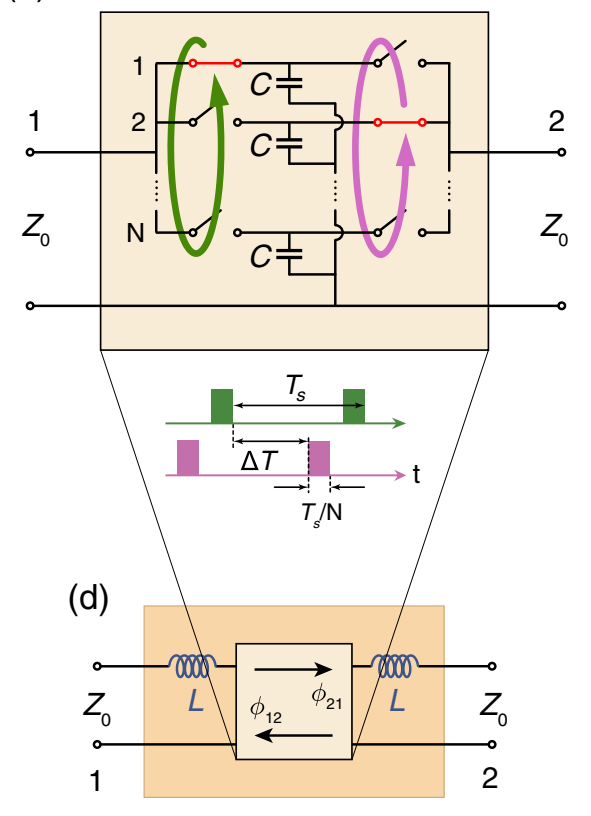

(b)

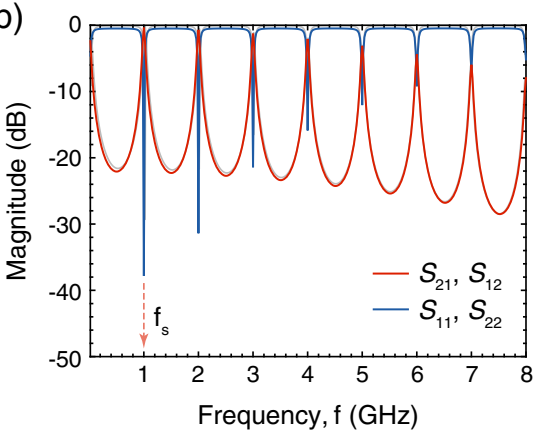

(e)

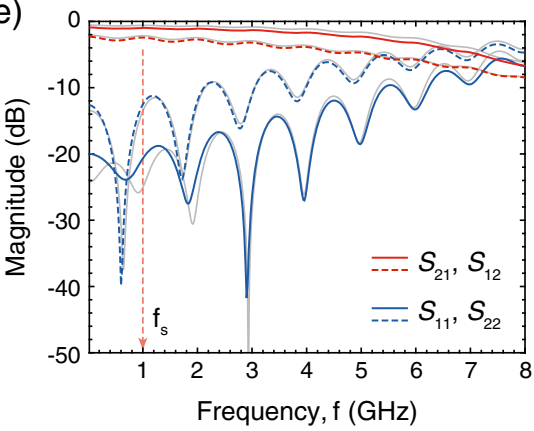

(c)

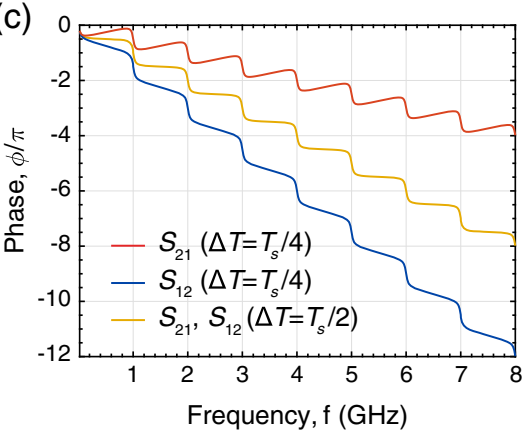

(f)

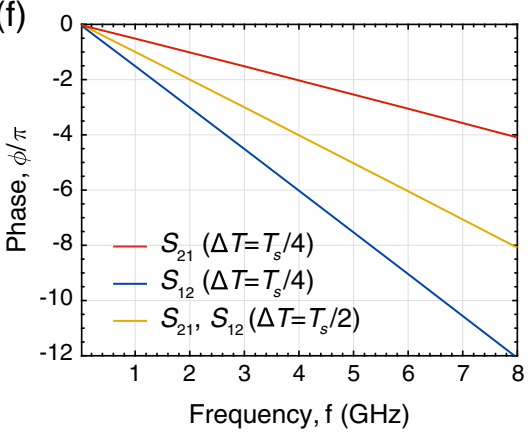

FIG. 1. (a) Schematic of an electronic circuit with $N$ identical paths containing switched shunt capacitances. Each switch is closed over the interval $T_{s} / N$, where $T_{s}$ is the switching period, and the input and output set of switches are operated in a revolving fashion. The output set lags the input set by time $\Delta T$ corresponding to a phase shift $\gamma=-2 \pi \Delta T / T_{s}$. (b),(c) Magnitude and phase of the scattering parameters of the network in (a) with $N=16, C=15 \mathrm{pF}, f_{s}=1 \mathrm{GHz}$, and $Z_{0}=50 \Omega$, leading to $\tau \gg T_{s} / N$ (here, $\tau=Z_{0} C$ ), which allows it to operate as an $N$-path filter. The phase response is computed for $\Delta T=T_{s} / 2$ (phase-reciprocal case) and $\Delta T=T_{s} / 4$ (phase-nonreciprocal case). For both cases, the magnitudes of scattering parameters are identical. (d) Schematic of a circuit operating as a wideband phase shifter, comprised of a commutated network with small series inductances on both sides. (e),(f) The same as (b),(c), but with $C=0.68 \mathrm{pF}$, leading to $\tau \sim T_{s} / N$, which allows the network to operate as a wideband phase shifter. Dashed lines and solid lines show the response with and without small matching inductances $L=0.75 \mathrm{nH}$, respectively. (f) Corresponding phase response. Results in (b), (c), (e), and (f) are obtained by means of the method outlined in the Appendix. The light gray "shadow" curves in (b) and (e) indicate simulation results obtained using Cadence Spectre RF (PSS solver) [47]. 
depends inversely on $\tau$ : The larger the time constant, the narrower the transmission peaks. For this reason, $\mathrm{N}$-path filters have become attractive alternatives to conventional filters for high- $Q$ applications, with the additional inherent advantages of reconfigurability and full compatibility with CMOS integrated circuit technology. Recently, it has been shown that $N$-path filters can also exhibit both reciprocal and nonreciprocal phase responses depending on the delay $\Delta T$ between the two sets of switches [40,45]. To illustrate this aspect, in Figs. 1(b) and 1(c), we show amplitudes and phases of scattering parameters $S_{i j}=$ $\left|S_{i j}\right| e^{j \phi_{i j}}$ of an $N$-path filter for two different switching delays, $\Delta T=T_{s} / 4$ and $\Delta T=T_{s} / 2$, corresponding to commutation phase shifts $\gamma=-\pi / 2$ and $\gamma=-\pi$, respectively. It is seen that the transmission phases are generally nonreciprocal: At $\omega=m \omega_{s}$ (here, $\omega=2 \pi f$ is the angular frequency), the corresponding phases are $\phi_{21}\left(m \omega_{s}\right)=m \gamma$ and $\phi_{12}\left(m \omega_{s}\right)=-m(2 \pi+\gamma)$. For $\Delta T=T_{s} / 2$, the network is fully reciprocal, as seen in Fig. 1(c) [49]. It is important to stress that the transmission peaks and phase shifts depend solely on $f_{s}$ and $\gamma$; i.e., such commutated networks break the dependence of the phase delay on the constituent elements, in this case the time constant. This property makes commutated networks interesting candidates to obtain large phase shifts in small footprints. Their small bandwidths, however, limit their use as conventional delay lines.

In the opposite limit, $\tau \ll T_{s} / N$, such commutated network operates as a track-and-hold circuit. When the input switch is closed, the capacitor voltage tracks the input voltage and stores its final value. Then, when the output switch is closed, this value is released to the output port in the form of a Dirac delta-function-like voltage spike. Such track-andhold functionality is routinely employed in analog-to-digital converters [50].

In this work, we employ an unexplored regime of commutated network operation, $\tau \sim T_{s} / N$. Contrary to the filtering functionality achieved when $\tau \gg T_{s} / N$ and the sampling functionality obtained for $\tau \ll T_{S} / N$, in this case, the network operates as an extremely wideband phase shifter with low insertion loss. To improve impedance matching to $Z_{0}$, we add two small inductances $L$ on either side of the network, as shown in Fig. 1(d). In Fig. 1(e), we plot the magnitude of the scattering parameters with $N=16, C=0.68 \mathrm{pF}$, with and without the presence of small series inductances $L=0.75 \mathrm{nH}$. The addition of small inductances improves the insertion loss by approximately $2 \mathrm{~dB}$ and return loss by approximately $10 \mathrm{~dB}$. The corresponding phases $\phi_{21}(\omega)$ and $\phi_{12}(\omega)$ are plotted in Fig. 1(f). It is seen that the phase response is ideally linear over an extremely broad bandwidth, and the phase slopes depend only on the commutation phase shift $\gamma$ as

$$
\phi_{21}(\omega)=\gamma \frac{\omega}{\omega_{s}},
$$

$$
\phi_{12}(\omega)=-(2 \pi+\gamma) \frac{\omega}{\omega_{s}} .
$$

To fully understand the nature of such a drastic change of the network response, we perform time- and frequencydomain analyses of the signal transmission. In the frequency domain, each switch operates periodically with frequency $f_{s}$ (in what follows, $f_{s}=1 \mathrm{GHz}$ ) and acts as a frequency mixer converting the monochromatic input signal at some frequency $f_{0}$ into a sum of discrete harmonics oscillating at frequencies $f_{0}+n f_{s}$ with $n=$ $0, \pm 1, \pm 2, \ldots$. The shunt capacitance $C$ in each branch is a low-pass filter, whose bandwidth is determined by the $R C$ constant $\tau=Z_{0} C$. In Fig. 2, we plot its scattering parameters for the two cases discussed in Fig. 1, i.e., $C=15 \mathrm{pF}$ and $C=0.68 \mathrm{pF}$ (for both cases, $Z_{0}=50 \Omega$ ). From Fig. 2(a), it is seen that for $C=15 \mathrm{pF}$ the shunt capacitor filters all positive- and negative-frequency harmonics impinging from port 1 except, possibly, the one near dc. This leads to a series of narrow peaks in the transmission of the commutated network at frequencies $m f_{s}, m=$ $0,1,2, \ldots$ [see Fig. 1(b)], which correspond to transmission of the harmonics $n=0,-1,-2, \ldots$, respectively. From Fig. 2(b), it is seen that the phase $\phi_{21}$ in this case exhibits a rapid variation, owing to the proximity of the pole $\omega_{\text {pole }}=$ $j \tau^{-1}$ to the origin of the complex frequency plane, leading to a staircase phase profile of the commutated network, see Fig. 1(c). On the other hand, for $C=0.68 \mathrm{pF}$ (see dashed lines in Fig. 2), the pole moves further away from the origin, allowing multiple harmonics to be transmitted at
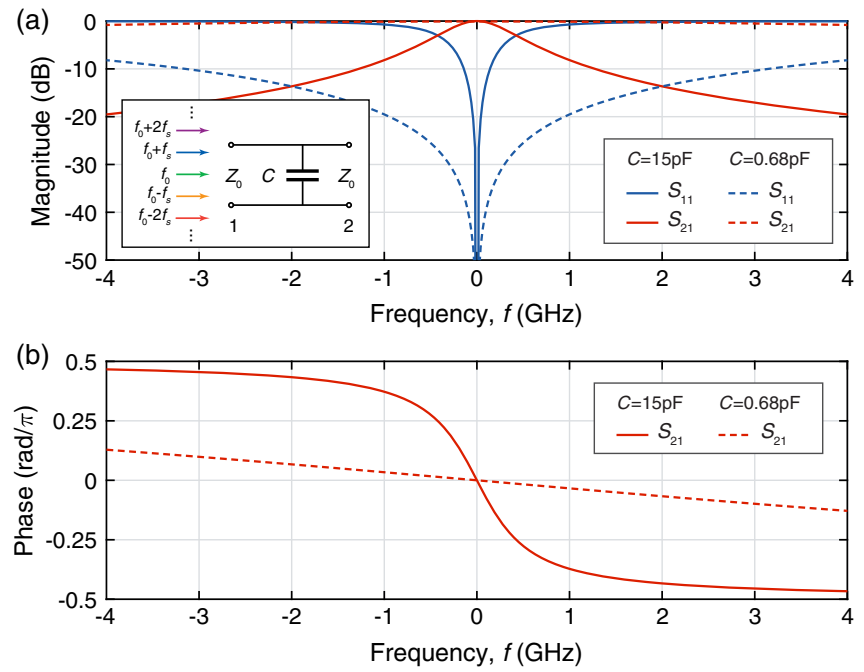

FIG. 2. (a) Amplitude of the scattering parameters of the shunt capacitance $C$ shown in the inset as a function of the frequency (with negative frequencies included). The reference impedance is $Z_{0}=50 \Omega$. The inset shows the shunt capacitor network being excited by a multitone signal containing only a discrete set of harmonics $f_{0}+n f_{s}$ with $n=0, \pm 1, \pm 2, \ldots$ (b) Phases of the corresponding parameters $S_{21}$. 


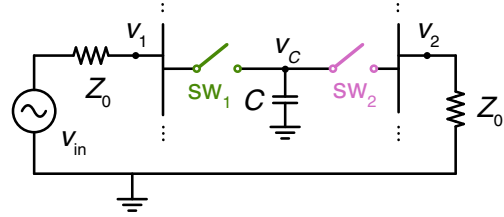

Key:
$\square v_{\text {in }} \quad$ sw $_{1}$ closed
$v_{1} \quad \mathrm{sw}_{2}$ closed
$v_{2}$
$v_{\mathrm{C}}$ (a)

(b)

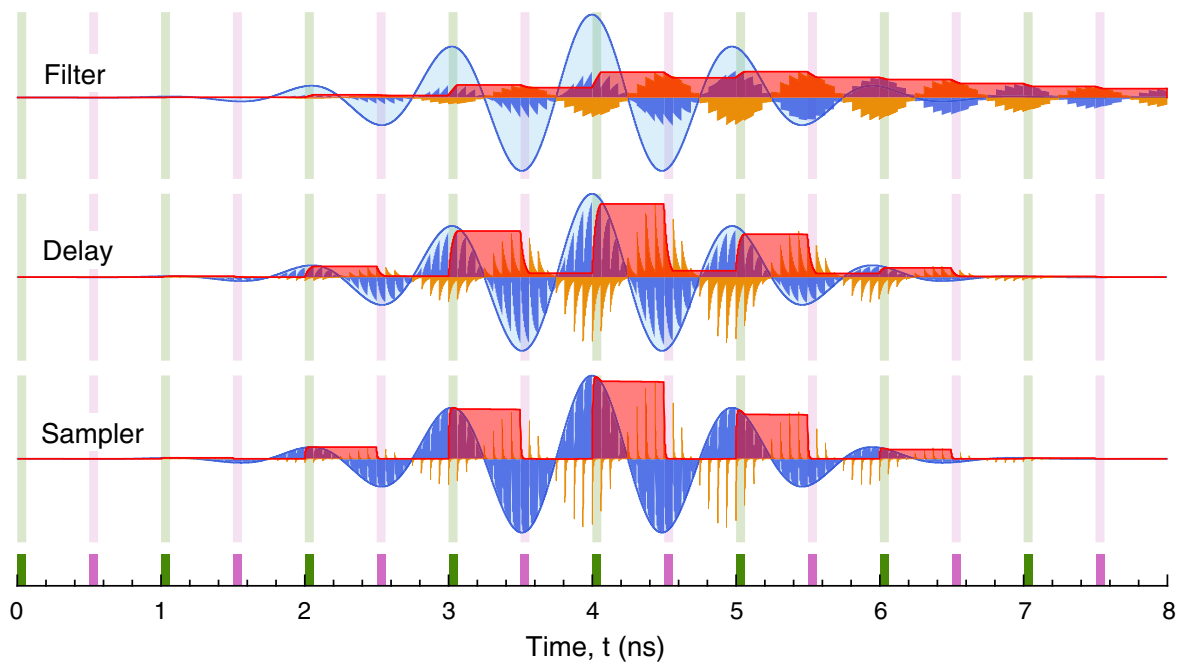

FIG. 3. Temporal evolution of the voltage across the capacitance, $v_{C}(t)$, in one branch of a commutated multipath network with $N=16, f_{s}=1 \mathrm{GHz}$, and $\Delta T=T_{s} / 2$, excited by a Gaussian pulse with the center frequency $f_{0}=1 \mathrm{GHz}$ and a duration of $4 \mathrm{~ns}$. The tree cases shown correspond to (a) $C=5 \mathrm{pF}$, at which the network operates as a high- $Q$ filter, (b) $C=0.5 \mathrm{pF}$, when the network acts as a broadband delay element, and (c) $C=0.1 \mathrm{pF}$, for which the circuit operates a sampling network. Green and pink bars indicate time intervals when the input and output switches, respectively, in the first branch are closed. The analysis is performed using Keysight ADS [51].

port 2 , hence resulting in a nearly flat phase response. This property leads to a wideband transmission of the commutated network and linearization of its phase profile.

In Fig. 3, we plot the temporal evolution of the voltage in one of the capacitors $v_{C}(t)$, when the network is excited by a Gaussian pulse with the center frequency $f_{0}=f_{s}$. When the shunt capacitance is large, $\tau \gg T_{s} / N$ [see Fig. 3(a)], we are in the filtering regime in which $v_{C}$ cannot faithfully follow the input voltage, because the charging time of the capacitor is much longer than the time over which the switch is closed. For the same reason, it cannot discharge fast enough to recreate the input signal at the output port. The signal is transmitted only for $f=m f_{s}$ with $m=1,2, \ldots$, because only at these frequencies does the input signal share the same period as the clock signal, allowing each capacitor to sample the same input voltage at every cycle.

On the other hand, when $\tau \sim T_{s} / N$ [see Fig. 3(b)], the capacitor voltage $v_{C}$ almost reaches the input voltage during the time over which the input switch is closed. Similarly, when the output switch is closed, the capacitor has enough time to discharge. The staggered storage and release of portions of the signals in each capacitive branch allows imparting very large time delays, independent of the frequency of operation. Therefore, such a regime enables extremely large bandwidths and allows the pulse to propagate without distortion. The insertion loss is explained by the excitation of intermodulation products caused by large voltage sweeps in each branch when the switches are closed, which are reflected as small spikes visible in the transmitted pulse but which do not distort its envelope. The transmission properties gradually deteriorate as the frequency increases, as seen in Fig. 1(e), consistent with the Nyquist-Shannon theorem [52] which establishes the relation between the sampling frequency and the highest frequency $f_{\max }$ beyond which the aliasing and frequency folding will occur. In our case, the sampling frequency is $N f_{s}$ corresponding to $f_{\max }<N f_{s} / 2$, which is about $8 \mathrm{GHz}$ in the current example, in agreement with Fig. 1(e). The available bandwidth can be straightforwardly broadened by increasing the number of paths in the commutated network.

Finally, in Fig. 3(c), we plot the capacitor voltage when the network is in the sampling regime $\tau \ll T_{s} / N$. In this case, for most of the time $v_{1}(t) \approx v_{\text {in }}(t)$;i.e., the network is an open circuit reflecting the input signal back to the source, because almost no net current is flowing through the source resistance. In turn, the output voltage comprises a series of narrow delta-function-like voltage spikes whose amplitudes are determined by the capacitor voltage immediately before the corresponding input switch opens (thus, the name "track-and-hold" circuit).

The transition of the spectral response of the network between filtering, analog delay, and sampling regimes is summarized in Fig. 4, in which we plot the insertion and return loss versus the frequency and time constant $\tau$. We also indicate the three representative values of $\tau$ corresponding to each of the three cases discussed in Fig. 3. It is seen that, with a decrease of $\tau$, the network experiences a gradual transition from filtering to the low-loss wideband delay functionality of interest here. We also note that, due to this smooth transition, there is no well-defined optimal value of $\tau$. In loose terms, it is the value for which the peaks in $S_{11}$ and $S_{21}$ disappear and the corresponding phases become linear. With a further decrease in $\tau$, the network 
(a)

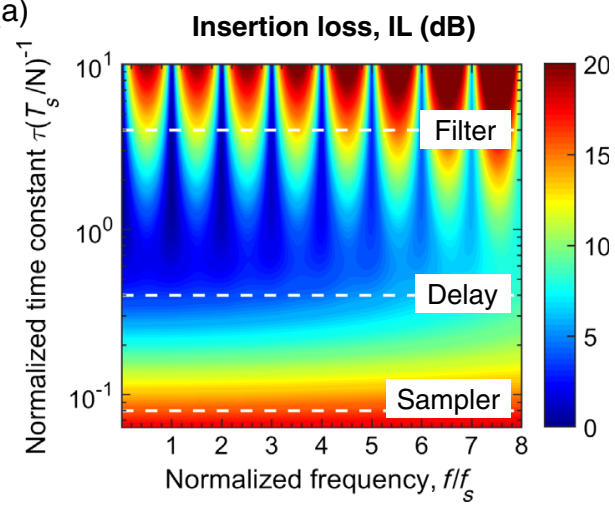

(b)

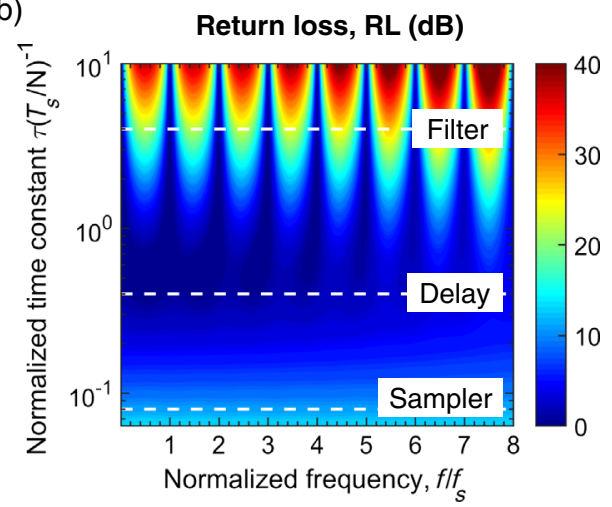

FIG. 4. (a) Insertion loss $\mathrm{IL}=-20 \log _{10}\left|S_{21}\right| \mathrm{dB}$ and (b) return loss $\mathrm{RL}=-10 \log _{10}\left(1-\left|S_{11}\right|^{2}\right) \mathrm{dB}$ of the commutated capacitor network shown in Fig. 1(a) versus the normalized frequency $f / f_{s}\left(f_{s}\right.$ is the modulation frequency) and the $R C$ time constant $\tau=Z_{0} C$ normalized by the time $T_{s} / N$ over which each switch remains closed. The three dashed horizontal lines indicate the three representative values of $\tau$ for $C=5 \mathrm{pF}, C=0.5 \mathrm{pF}$, and $C=0.1 \mathrm{pF}$ corresponding to network operating, respectively, as a filter, a delay element, and a sampler. All other parameters are the same as in Fig. 1.

enters the sampling regime, also wideband, but with a much higher insertion and return loss.

\section{WAVE PROPAGATION IN PERIODIC NETWORKS OF COMMUTATED CAPACITORS}

Having introduced the operation principles of a single commutated multipath network, here we study wave propagation through a cascade of such networks. It is well known that time-invariant periodic networks consisting of static $L$ and $C$ support passbands and stop bands. Their cutoff frequencies and phase and group velocities are defined by the values of $L$ and $C$ and the spatial periodicity. On the contrary, here we show that periodic networks of commutated capacitors fundamentally break this dependence and that their phase and group velocities depend only on the commutation phase shift $\gamma$ and modulation frequency $f_{s}$, opening exciting opportunities to realize electrically small slow-wave structures with unbounded DBPs.

Consider an infinite cascade of commutated wideband phase-shifting networks. Since the insertion loss and intermodulation products are fairly small, we can approximate the response of a unit cell by the scattering matrix of a lossless nonreciprocal time-invariant phase shifter:

$$
\mathbf{S} \approx\left[\begin{array}{cc}
0 & e^{j \phi_{12}} \\
e^{j \phi_{21}} & 0
\end{array}\right]
$$

The two solutions for Bloch phase shifts [53] over a single unit cell, $k a \in[-\pi, \pi]$ ( $k$ and $a$ denote, respectively, the Bloch wave number and the unit-cell size), can be found using Eqs. (1) and (2):

$$
k_{1}(\omega) a=-\phi_{21}(\omega)=-\gamma \frac{\omega}{\omega_{s}},
$$

$$
k_{2}(\omega) a=\phi_{12}(\omega)=-(2 \pi+\gamma) \frac{\omega}{\omega_{s}} .
$$

To verify this result, we compare it against rigorous numerical solutions of a multifrequency periodic problem (see the Appendix) in the two cases: reciprocal, $\Delta T=T_{s} / 2$ $(\gamma=-\pi)$, shown in Fig. 5(a), and nonreciprocal, $\Delta T=$ $(N-1) T_{s} / N$ corresponding to $\gamma=-2 \pi(N-1) / N$, shown in Fig. 5(b). It is seen that in both cases analytical and numerical solutions closely agree, showing a linear and gapless dispersion. Small discrepancies between the results stem from the small inductances $L$, which, however, do not introduce significant changes to the network dispersion. Nevertheless, including these small inductances between commutated networks is required to sustain signal propagation: Through these inductances, the capacitors can charge and discharge, and they enable improved impedance matching to $Z_{0}$.

In Figs. 5(a) and 5(b), the networks have identical bandwidths, but in the reciprocal case the group velocity $v_{g}=\partial \omega / \partial k$ is the same in both directions,

$$
v_{g}^{ \pm}= \pm 2 a f_{s},
$$

while in the nonreciprocal case

$$
\begin{gathered}
v_{g}^{+}=\frac{N}{N-1} a f_{s}, \\
v_{g}^{-}=-a N f_{s} .
\end{gathered}
$$

Given the number of paths $N$ and the commutation frequency $f_{s}$, Eqs. (7) and (8) provide the lower and upper bounds for group velocity achievable in such commutated networks. From these expressions, it is evident that the group velocity can be made arbitrarily small by simply reducing the commutation frequency $f_{s}$. The bandwidth 

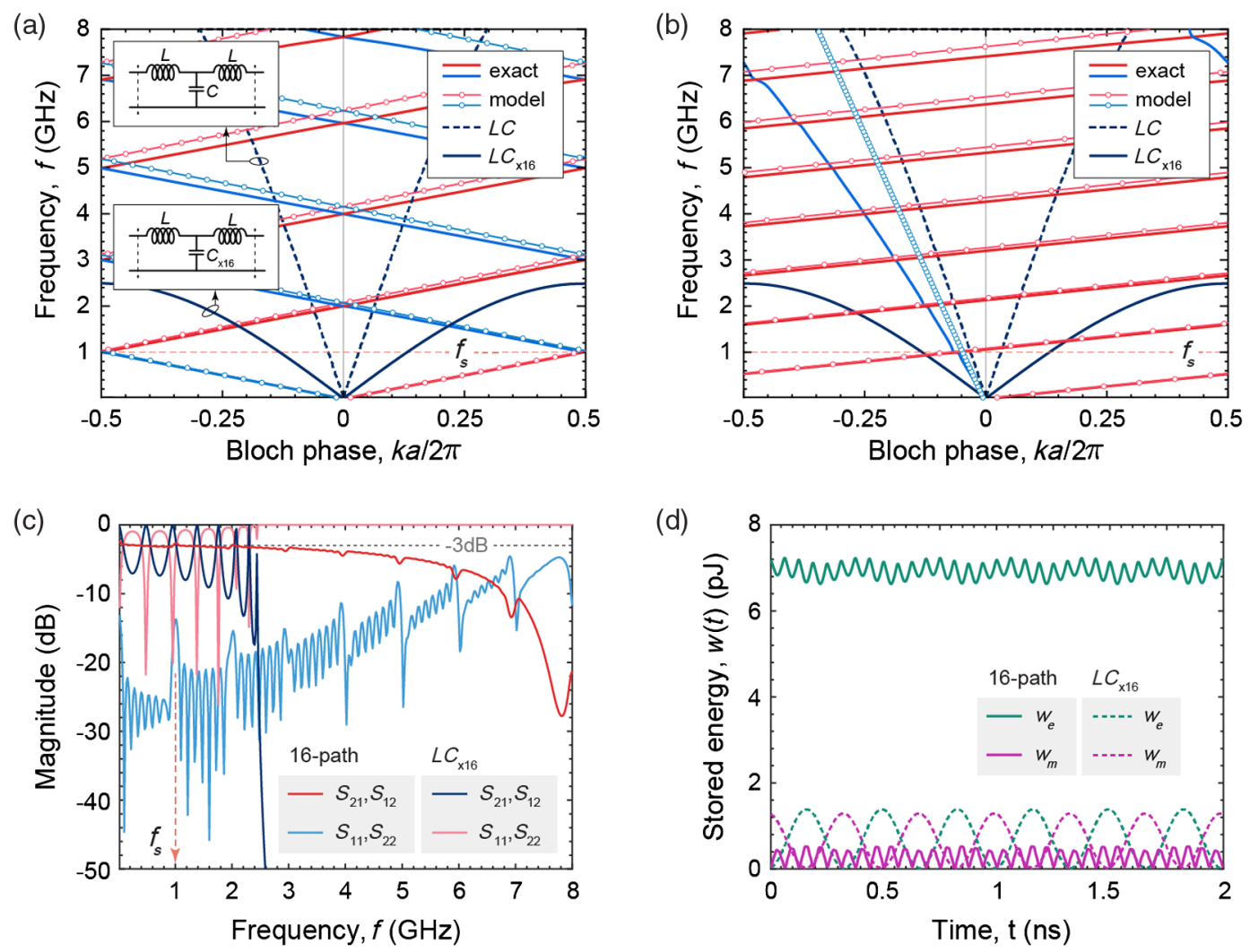

FIG. 5. (a) Band diagram of cascaded commutated networks shown in Figs. 1(d)-1(f) computed for $\Delta T=T_{s} / 2$, for which the network is reciprocal at all frequencies [all other parameters are taken to be the same as in Figs. 1(d)-1(f) with matching inductors included]. Numerical results (bold lines) are obtained using the method outlined in the Appendix. Thin lines with markers indicate an idealized nonreciprocal phase shifter response computed by means of Eqs. (4) and (5). A small discrepancy arises from the additional phase shift provided by the small inductances. Dark blue solid and dashed lines indicate Bloch phase shifts of time-invariant periodic $L C$ transmission lines with per-period capacitance $C=0.68 \mathrm{pF}$ and $C_{\times 16}=16 C$, respectively. (b) The same as in (a), but for $\Delta T=$ $15 T_{s} / 16$ corresponding to maximum phase nonreciprocity. (c) Comparison between the scattering parameters of eight cascaded unit cells of the commutated network and a time-invariant $L C$ delay line. Each unit cell of the $L C$ delay line has the same total inductance $2 L$ and capacitance $C_{\times 16}$ as the commutated network. (d) Total stored electric and magnetic energy at $1.5 \mathrm{GHz}$ (steady state) in eight cascaded commutated networks (solid lines) and $L C$ delay line (dashed lines) with equal total capacitance and inductance.

$\Delta f$ is independent of the group velocity, and it is limited only by the Nyquist-Shannon theorem: $\Delta f<N f_{s} / 2$.

To further emphasize the broadband slow-wave effect enabled by commutation, it is instructive to compare the band diagrams of time-varying and time-invariant networks. In Figs. 5(a) and 5(b), we plot the band diagrams of time-invariant transmission lines composed of $L C L$ sections with the same values of $L$ and $C$ as a single branch of the commutated network (see the dashed dark blue lines). This comparison is reasonable, since at any instant of time the signal sees just one of the branches of the multipath network, while all other switches are open. It is seen that in this case the group velocity is much larger, while only a small phase shift is accumulated over one unit cell. The latter also represents the minimal phase shift that can be obtained in the commutated multipath network; i.e., it is the "light cone" of the system. In both panels, we also plot the dispersion of a "high-index" $L C L$-transmission line with the capacitance $C_{\times 16}=16 C$, i.e., with the total $L$ and $C$ equal to those of the commutated network when all switches are permanently closed, so that all the capacitances become connected in parallel. The group velocity in this case is lower than in the single branch case, as expected, at the expense of a significantly diminished bandwidth. Very interestingly, the group velocity of the commutated network is still much smaller than that of the high-index transmission line, and it has essentially no dispersion over the entire frequency range. In addition, it can be made larger or smaller than the one of a high-index transmission line by simply changing the modulation frequency $f_{s}$ and the switching delay $\Delta T$. By increasing the number of paths $N$ for a fixed modulation frequency, we obtain smaller and smaller required $C$ to maintain the relation $Z_{0} C \sim T_{s} / N$ and also smaller $L$ required to achieve good impedance matching to $Z_{0}$, potentially allowing for an arbitrarily large contrast between the effective indexes of static and commutated networks. 
In Fig. 5(c), we plot the scattering parameters of a commutated network comprising $K=8$ unit cells. Such a network accumulates a total insertion loss of about $3 \mathrm{~dB}$ with good impedance matching to $Z_{0}$ over a very large bandwidth. Expectedly, the transmission properties deteriorate close to $8 \mathrm{GHz}$, in agreement with the NyquistShannon theorem. In the same panel, we also plot the scattering parameters of a time-invariant high-index transmission line composed of the same number of cascaded $L C L$ segments. As one may expect, besides the appearance of a band gap at about $2.5 \mathrm{GHz}$, the high-index line is not matched to $Z_{0}$, resulting in a series of narrow transmission peaks.

At the core of the remarkable differences between a conventional high-index line and our commutated network is the inherent imbalance between electric and magnetic stored energy in the system. While any time-invariant waveguide supports wave propagation by exchanging in time and space equal amounts of energy stored in electric and magnetic forms, wave propagation in our commutated network is quasielectrostatic in nature, residing for the most part in the capacitive banks at any instant in time. In fact, it is possible to achieve an arbitrarily large ratio between stored electric and magnetic energy by simply increasing the number of paths $N$. To better illustrate this property, Fig. 5(d) shows the temporal evolution of the total electric and magnetic energies stored in the eight-unit-cell commutated and high-index time-invariant networks in Fig. 5(c) in a steady state at $f=1.5 \mathrm{GHz}$ for which both networks are matched to the source and load impedance $Z_{0}$. In contrast to the static network, which exhibits periodic oscillations of equal amounts of electric and magnetic stored energies $w_{e}$ and $w_{m}$, respectively, it is seen that in the commutated network $w_{e} \gg w_{m}$. We also notice a much larger total stored energy in the commutated network, which is consistent with the ultrabroadband slowdown of the power flow along the waveguide. Clearly, the commutated network unveils a new form of wave propagation, which enables uniquely broadband dispersion-free slowwave propagation beyond the typical delay-bandwidth trade-off.

In comparison, another well-known delay system that stores energy in a purely electrostatic form is a distributed $R C$ delay line formed by a ladder of $R C$ filters whose time constant is chosen to be small enough to obtain an ideally linear and broadband phase shift, much like the commutated network presented here but without temporal modulations. Because of their extremely compact footprint, $R C$ delay lines are compatible with on-chip integration [54]. However, the dynamic of $R C$ delay lines is governed by the diffusion equation [55]; i.e., they do not sustain wave propagation, translating into very high insertion loss and impedance mismatch. In this paper, we show that suitable temporal modulation allows overcoming this issue by restoring wave propagation while preserving the wideband linear phase response, with the additional benefit of dynamic reconfigurability.

Since the bandwidth of the commutated network is limited only by the Nyquist-Shannon sampling theorem $\Delta f \sim N f_{s} / 2$, we can estimate the maximal achievable DBP in a network with $K$ unit cells. In the reciprocal case, from Eq. (6) we obtain

$$
\Delta t \cdot \Delta f \sim \frac{K a}{2 a f_{s}} \cdot \frac{N f_{s}}{2}=K \frac{N}{4} .
$$

In turn, the largest DBP for transmission from port 1 to port 2 is obtained from the condition for maximum phase nonreciprocity (7):

$$
\Delta t_{21} \cdot \Delta f \sim \frac{K a(N-1)}{a N f_{s}} \cdot \frac{N f_{s}}{2}=K \frac{N-1}{2},
$$

while in the reverse direction it is

$$
\Delta t_{12} \cdot \Delta f \sim \frac{K a}{a N f_{s}} \cdot \frac{N f_{s}}{2}=\frac{K}{2} .
$$

For all cases, the DBP for the round trip is $\left(\Delta t_{21}+\Delta t_{12}\right)$. $\Delta f \sim K N / 2$ [48]. From Eqs. (9) and (10), it is seen that, by increasing the number of paths, it is possible to achieve an arbitrarily large DBP within a limited footprint, thanks to the fact that most of the energy is stored in electric form in capacitor banks, which are amenable to extremely compact integration in CMOS technology.

It is also instructive to compare the DBP of the commutated network with the DBP of a time-invariant $L C L$ delay line with the same number of unit cells $K$. Assuming no impedance mismatch, the available bandwidth $\Delta f$ can be roughly estimated as $\Delta f \sim 2 v_{g} / a$, where $a$ is the physical length of the unit cell (assuming one single-mode resonator per cell) [12]. For the group delay, we have $\Delta \tau \sim K a / v_{g}$. Thus, the DBP of a time-invariant $L C L$ line is $\Delta \tau \cdot \Delta f \sim 2 K$, clearly showing that the commutated network can overcome this limit. This result opens truly remarkable opportunities to achieve ultrabroadband slow-wave regimes in a platform compatible with modern integrated circuits. We stress that the unit cells considered here can be directly implemented in CMOS integrated circuits by simply using the well-established $N$-path filter technology and modifying the switching strategy.

In this context, another important advantage of the proposed commutated network consists in the fact that, in contrast to other spatiotemporally modulated systems that require accurate and coherent modulation of the local properties of carrier media or spatially distinct elements, here the modulation is applied locally in each cell and, thus, only switches within the same unit cell must be operated synchronously, which can be easily achieved by embedding an independent clock generator within each 
element. In addition, these networks employ only two states of switches, ON and OFF, leading to significantly enhanced linearity to the amplitude of the input signal [40]another important advantage over harmonically modulated systems that generally require the modulation signal to be much stronger than the input signal.

The fact that commutated networks can operate as TTDs (i.e., analog signal buffers) can be appreciated in Fig. 6, where we show three wideband pulses with different center frequencies $f_{0}$ propagating through a commutated network comprising eight unit cells with a switching delay $\Delta T=$ $(N-1) T_{s} / N=15 T_{s} / 16$. All three pulses have the same relative FWHM $=2 \sqrt{2 \ln 2} f_{0}^{-1}$. It is seen that their widths remain unchanged, due to the near-zero group velocity dispersion. The temporal delay for propagation from port 1 to port 2 is also the same, $\Delta t_{21}=K \Delta T=7.5 \mathrm{~ns}$, while in the reversed direction $\Delta t_{12}=K\left(T_{s}-\Delta T\right)=0.5 \mathrm{~ns}$, yielding an extremely broadband nonreciprocal delay. As discussed above, insertion loss and amplitude reduction are primarily associated with the excitation of high-frequency intermodulation products manifested by the spikes present in the transmitted pulses. It is worth noting, however, that these intermodulation products occur only at high frequencies $f_{0}+m N f_{s}, m=1,2,3, \ldots[40,45]$, which can be filtered out. This property is confirmed in Fig. 6, in which,

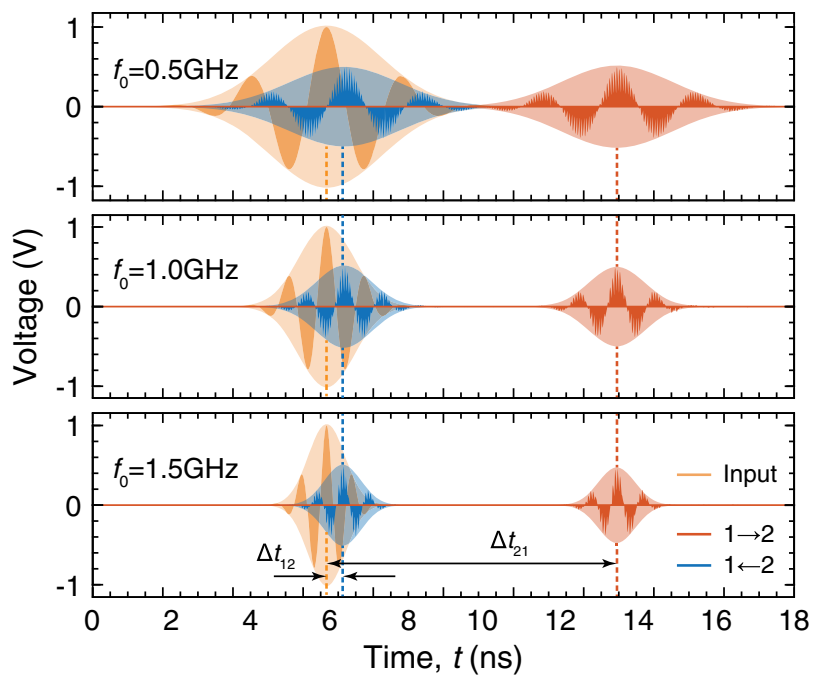

FIG. 6. Propagation of pulses with center frequency $f_{0}=0.5$, 1.0, and $1.5 \mathrm{GHz}$ through an eight-unit-cell cascaded commutated network with $N=16, C=0.68 \mathrm{pF}, L=0.75 \mathrm{nH}$, and switching delay $\Delta T=15 T_{s} / 16$. The FWHM duration of each input pulse (yellow lines) is $2 \sqrt{2 \ln 2} f_{0}^{-1}$. For pulses sent from port 1 to port 2 (red lines), the accumulated time delay is $\Delta t_{21}=8 \Delta T$. For pulses sent from port 2 to port 1 (blue lines), the time delay is $\Delta t_{12}=8 T_{s}-\Delta t_{21}$. The width of the forward and reverse pulses remains unchanged due to nearly zero group velocity dispersion. The analysis is performed using Keysight ADS (time-domain analysis) [51]. despite the spikes, the overall shape of the pulses is fully preserved.

\section{APPLICATION TO PRACTICAL DEVICES}

Having established the principles of slow-wave propagation in time-modulated multipath networks, in this section we apply them to design highly miniaturized reciprocal and nonreciprocal microwave devices of practical relevance. When $\gamma=-\pi$, the switching scheme is symmetric. Hence, the commutated networks are inherently reciprocal over the entire frequency range, $\phi_{21}(\omega)=$ $\phi_{12}(\omega)=-\pi \omega / \omega_{s}$. This regime can be used to realize reciprocal phase shifters with arbitrarily large delay-bandwidth products. For example, a phase shift $\phi_{21}=\phi_{12}=$ $-m \pi / 2$ with $m=1,2,3, \ldots$ is achieved at frequencies $f_{0}=m f_{s} / 2$. From Eqs. (1) and (2), it also follows that the same reciprocal phase shifts can be achieved at frequencies $f_{0}=(m+2 n) f_{s} / 2$ with $n=0,1,2, \ldots$ if we use $\gamma=-m \pi /(m+2 n)$, which corresponds to an asymmetric commutation scheme yet provides a reciprocal response.

Nonreciprocal phase responses are obtained with asymmetric phase shifts. For instance, $\phi_{21}=-m \pi / 2$ and $\phi_{12}=$ $-(2 \pi-m \pi / 2)$ can be realized at frequencies $f_{0}=m f_{s}$, where $m=1,2,3, \ldots$. The available phase response of a standalone commutated network is summarized in Fig. 7, in which red and green markers indicate, respectively, nonreciprocal and reciprocal phase delays which are integer multiples of $-\pi / 2$. The shaded area indicates the range of phases accessible within the same device by simply changing the modulation scheme. Nonreciprocal markers appear in pairs: If the phase in one direction corresponds to one of the two red markers, its counterpart indicates the phase in

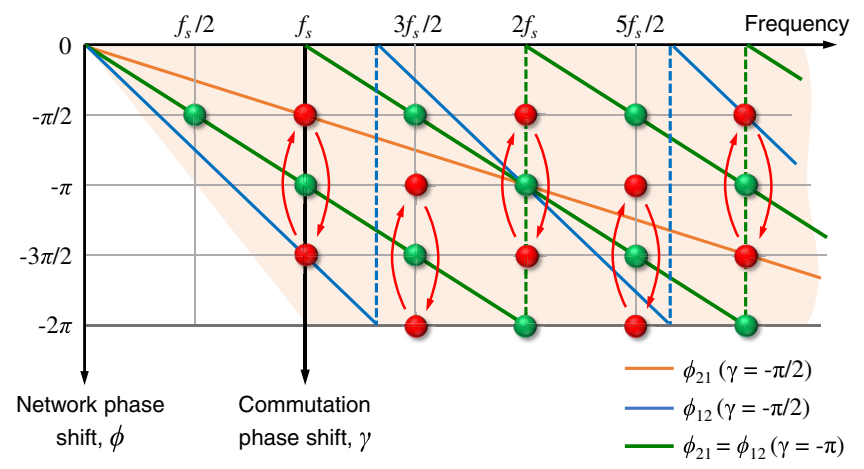

FIG. 7. Achievable phase shifts in the multipath network shown in Fig. 1 as a function of the commutation phase shift $\gamma$ and commutation frequency $f_{s}$, for $\tau \sim T_{s} / N$. Green and red markers indicate reciprocal and nonreciprocal phase shifts, respectively, integer multiples of $-\pi / 2$. Red markers always appear in pairs, indicating phase shifts in opposite directions. The solid green line shows when the phases in two directions coalesce, leading to a reciprocal response. The light shaded area indicates the bounds of the achievable phase response. 
the opposite direction. Solid green lines show the relation between $\gamma$ and $f_{s}$ when the phases in the two directions match, leading to a reciprocal response. As an example, by selecting the commutation phase shift $\gamma=-\pi / 2$, we obtain $\phi_{21}=-\pi / 2$ and $\phi_{12}=-3 \pi / 2$ at $f=f_{s}, \phi_{21}=\phi_{12}=-\pi$ at $f=2 f_{s}, \phi_{21}=-3 \pi / 2$ and $\phi_{12}=-\pi / 2$ at $f=3 f_{s}$, and so on. Thus, it is seen that commutated networks exhibit an impressively rich response which can be dynamically reconfigured by simply changing $f_{s}$ and $\gamma$. Thanks to the large bandwidth, the commutation frequency can be substantially lower than $f_{0}$, as long as $f_{s}>2 f_{0} / N$.

\section{A. Miniaturized rat-race coupler}

Using Fig. 7, we can envision several realistic devices based on a single piece of hardware, just reconfiguring the commutation scheme. First, we target the design of a four-port rat-race (RR) coupler [53] operating at $f_{0}=$ $2.5 \mathrm{GHz}$ and comprising three $-\pi / 2$ and one $-3 \pi / 2$ reciprocal phase shifting segments with characteristic impedance $\sqrt{2} Z_{0}$ connected in a ring, as shown in Fig. 8(a). The functionality of this device implies that a signal sent from port 1 splits equally between ports 2 and 4 , while port 3 remains isolated. Typically, phase-shifting sections in these couplers are realized with transmission lines of appropriate lengths or using lumped $L C$ banks with
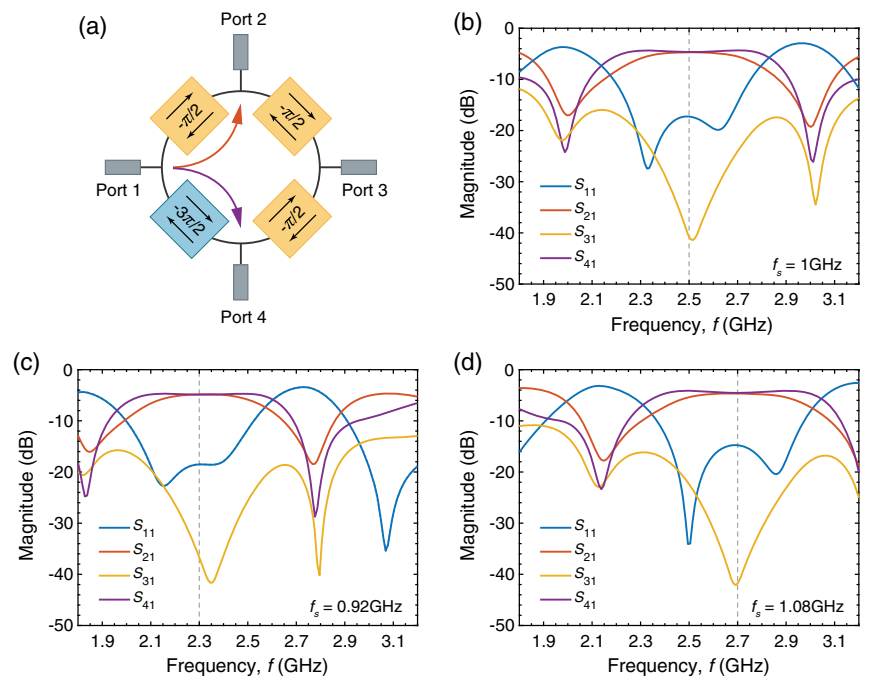

FIG. 8. (a) Schematic of a rat-race coupler aimed at $f_{0}=2.5 \mathrm{GHz}$, comprised of four commutated networks as in Fig. 1 , with $N=16, f_{s}=1 \mathrm{GHz}, C=0.47 \mathrm{pF}$, and $L=1 \mathrm{nH}$, connected in a ring. Three of the four sections are configured to provide a reciprocal phase shift $\phi_{21}=\phi_{12}=-\pi / 2$ at $f=f_{0}$ by choosing the switching delay $\Delta T=T_{s} / 2(\gamma=-\pi)$. The fourth section has $\Delta T=3 T_{s} / 10(\gamma=-3 \pi / 5)$, leading to $\phi_{21}=\phi_{12}=$ $-3 \pi / 2$ at $f=f_{0}$. (b) Corresponding scattering parameters computed using the numerical approach described in the Appendix. (c),(d) Scattering parameters for $f_{s}=0.92 \mathrm{GHz}$ and $f_{s}=1.08 \mathrm{GHz}$, respectively, with all other parameters unchanged.
$L \sim 7-10 \mathrm{nH}$. On-chip realization, therefore, is largely impractical due to the large occupied area and high loss of integrated inductors. On the contrary, our commutated networks require very little inductance, making these devices fully integrable.

From Fig. 7, it is seen that the two reciprocal phase shifts can be simultaneously achieved at $f_{0}=(2 m+1) f_{s} / 2$ with $m=1,2,3, \ldots$. To reduce power consumption and minimize the effects of timing errors, we choose $m=2$, which corresponds to $f_{s}=1 \mathrm{GHz}$. To obtain $-\pi / 2$ and $-3 \pi / 2$ phase shifts, we choose $\gamma=-\pi$ and $\gamma=-3 \pi / 5$, respectively. We stress the fact that the only difference between the $-\pi / 2$ and $-3 \pi / 2$ sections is the commutation phase shift, while all the circuitry is identical, making the practical implementation very appealing and reconfigurable in real time. The scattering parameters of the resulting network are shown in Fig. 8(b). It is seen that the proposed rat-race coupler provides a nearly perfect splitting of the signal incoming through port 1 between ports 2 and 4 and approximately $40 \mathrm{~dB}$ isolation at port 3 . The insertion loss of the coupler at the center frequency is $4.8 \mathrm{~dB}$, which is sufficiently close to the $3 \mathrm{~dB}$ insertion loss of an ideal lossless RR coupler. The $1.8 \mathrm{~dB}$ increase in the insertion loss is associated with the excitation of intermodulation products $[41,56]$. Impressively, the whole circuit is nearly inductorless and can be implemented on chip in a deeply subwavelength footprint [40]. Another unique feature of the proposed device is that any of the four commutated networks can be dynamically reconfigured to provide a $-\pi / 2$ or $-3 \pi / 2$ phase shift by only changing $\gamma$, so that any of the four ports can be dynamically configured to be an isolated port.

It is important to emphasize that, similar to conventional rat-race couplers, the bandwidth here is limited only by the different phase slopes of $-\pi / 2$ and $-3 \pi / 2$ sections. Since these phase shifts can be controlled through the commutation frequency, the latter can be used to dynamically tune the operation frequency. Figures 8(c) and 8(d) show the scattering parameters for the same device, but with $f_{s}=$ $0.92 \mathrm{GHz}$ and $f_{s}=1.08 \mathrm{GHz}$, corresponding to $f_{0}=$ $2.3 \mathrm{GHz}$ and $f_{0}=2.7 \mathrm{GHz}$, respectively. It is seen that all key metrics remain essentially the same. The only restriction on such reconfigurability comes from the fact that, far away from the initially designed $f_{s}$, the condition $\tau \sim T_{s} / N$ is not satisfied for the same value of capacitance. However, this issue can be also overcome if the value of the capacitance is retuned, for instance, using variable capacitors.

\section{B. Miniaturized circulator}

The fact that commutated networks provide nonreciprocal phase shifts has been successfully employed to realize a variety of magnetless nonreciprocal devices, such as circulators, gyrators, and isolators [40-44]. All these designs, however, rely on transmission-line sections 

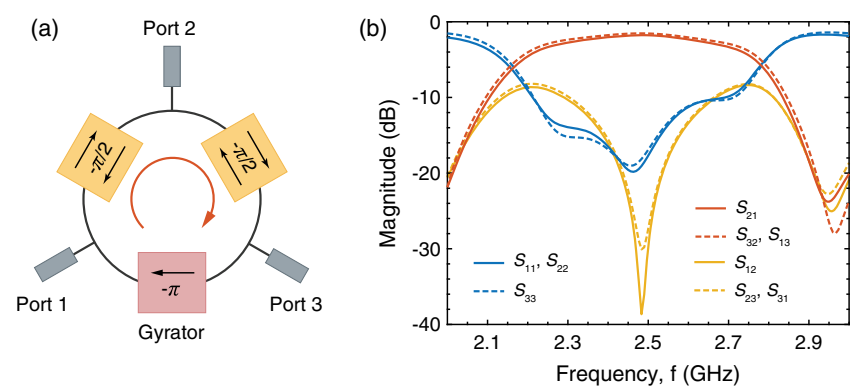

FIG. 9. (a) Schematic of a circulator operated at $f_{0}=2.5 \mathrm{GHz}$, comprised of three commutated networks as in Fig. 1, with $N=16, f_{s}=1 \mathrm{GHz}, C=0.65 \mathrm{pF}$, and $L=1 \mathrm{nH}$. Two of them are configured to provide a reciprocal phase shift $\phi_{21}=\phi_{12}=$ $-\pi / 2$ at $f=f_{0}$ by choosing the switching delay $\Delta T=T_{s} / 2$ $(\gamma=-\pi)$. The third network has $\Delta T=T_{s} / 5(\gamma=-2 \pi / 5)$, leading to a gyrator response $\phi_{21}=-\pi$ and $\phi_{12}=-2 \pi$ at $f=f_{0}$. (b) Corresponding scattering parameters computed using the numerical approach described in the Appendix.

or $L C$ tanks operating as delay lines to obtain required reciprocal phase shifts. Here, we propose the design of a miniaturized three-port circulator aimed at $f_{0}=2.5 \mathrm{GHz}$ employing commutated networks to replace both reciprocal and nonreciprocal sections. The circuit schematic is shown in Fig. 9(a): Two $-\pi / 2$ sections with the characteristic impedance $Z_{0}$ are wrapped around a gyrator providing a $-\pi$ phase shift in one direction. From Fig. 7, we find that the required phases can be achieved at $(2 m+1) f_{s} / 2$ with $m=1,2,3, \ldots$. Again, we choose $m=2$ corresponding to the modulation frequency $f_{s}=1 \mathrm{GHz}$. As in the case of a rat-race coupler, here we use $\gamma=-\pi$ for reciprocal $-\pi / 2$ sections. To obtain a gyrator, we choose $\gamma=-2 \pi / 5$, which leads to $\phi_{21}=-\pi$ and $\phi_{12}=-2 \pi$ at $f=f_{0}$. The resulting scattering parameters are shown in Fig. 9(b): The proposed network provides clockwise transmission and strong isolation in the counterclockwise direction. In principle, it is also possible to configure any of the three sections to act as a gyrator; however, the scattering parameters are already nearly symmetrical. At the center frequency, the circulator exhibits the insertion loss of around $2.5 \mathrm{~dB}$, on par with other magnetless circulator topologies [38-44], while being electrically a much smaller device. Similar to the rat-race coupler, the circulator bandwidth is limited only by different slopes of the phase profiles of reciprocal sections and the gyrator, and, therefore, the center frequency can be dynamically tuned by changing $f_{s}$.

\section{ADVANTAGES AND CHALLENGES OF PRACTICAL IMPLEMENTATION}

Throughout this work, the results are computed under the assumption of idealized switches having zero and infinite resistance in the ON and OFF state, respectively, a negligible parasitic capacitance, and zero rise and fall times for the modulation signals. In practice, in modern semiconductor technology, the ON switch resistance is on the order a few Ohms, and the rise and fall times are of the order of tens of picoseconds. Nonzero ON resistance and parasitic capacitance lead to a somewhat larger insertion loss but do not affect other key metrics of the network such as a large temporal delay and frequency-flat dispersion. The rise and fall times are typically small enough to not impact the performance at gigahertz-range modulation speeds discussed in this paper.

The use of switches provides significant advantages in terms of linearity and power handling, since switches tend to be more linear than analog varactors (variable capacitors, another commonly used approach for temporal modulation) and active approaches, such as active inductors [57]. Here, it is important to note that the $\mathrm{ON}$ resistance and linearity trade linearly with the power consumption in the modulation path: Larger switches with lower on resistance and, consequently, better linearity lead to linearly larger modulation power consumption. However, importantly, this trade-off improves with semiconductor technology scaling as semiconductor processes scale to finer dimensions, the parasitic capacitance and associated modulation power consumption reduce for a given $\mathrm{ON}$ resistance, improving loss and linearity without an increase in power consumption. This property stands in contrast to passive $L C$ circuits that exploit the metallization of semiconductor processes, the performance of which does not improve with technology scaling, and active approaches [57].

In our simulations, we also assume that the switch timing is ideal. For commutated multipath networks, it means that the only nonzero intermodulation products (higher-order harmonics) are those with $n=k N$, where $k= \pm 1, \pm 2, \ldots$. and $N$ is the number of paths [48]. In practice, small timing errors among the switches lead to all harmonics being nonzero and introducing some additional noise to the transmitted signal. However, this issue is common to all commutated networks, including $N$-path filters, which typically can achieve sufficiently low timing errors, on the order of a few percent at frequencies up to several gigahertz. Furthermore, timing errors can be corrected using calibration circuitry in the modulation path, as is commonly done in communication applications.

A small part of the noise at the signal frequency also converts to higher-order harmonics with $n=k N$, and noise at harmonic frequencies with $n=k N$ is converted back to the signal frequency. Noise folding from harmonic frequencies degrades the signal-to-noise ratio (SNR) of the input signal, and, as a result, the degradation in the SNR is larger than the insertion loss, unlike a conventional delay element realized using inductances and capacitance. However, this degradation can also be avoided by passing the input signal through a low pass filter with a cutoff frequency equal to the sampling frequency $N f_{s}$ to filter the noise at frequencies above the sampling frequency (commonly called an antialiasing filter). Also, the phase noise of the modulation 

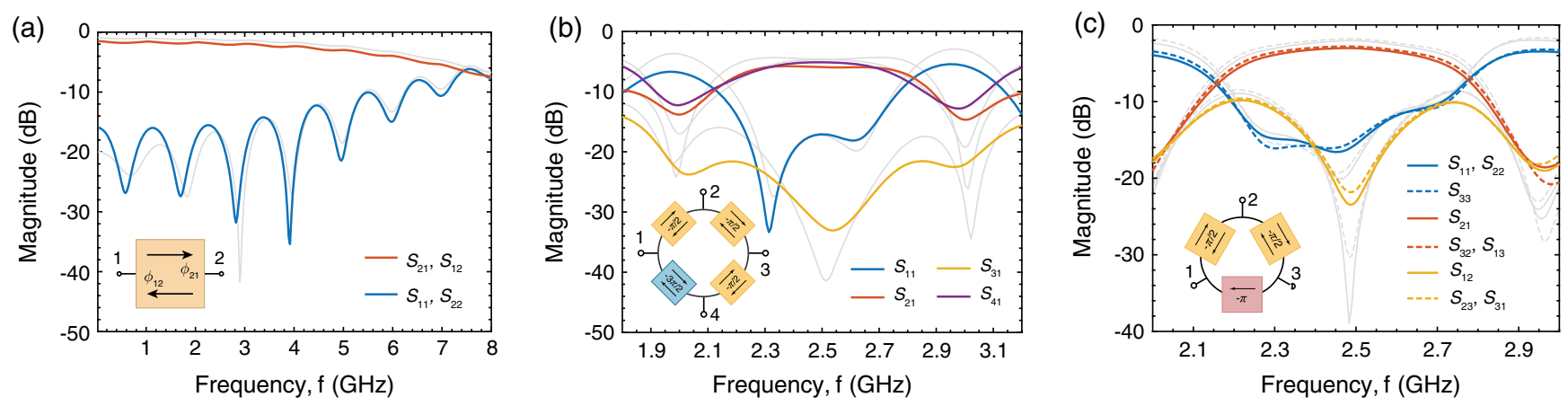

FIG. 10. The effect of realistic nonidealities of switches on the performance of the (a) commutated network, (b) rat-race coupler, and (c) circulator shown in Figs. 1(d) and 8(a), and 9(a), respectively. The on switch resistance is set to be $3 \Omega$, and the rise and fall times are $10 \mathrm{ps}$. We also include a random clocking error with a normal distribution of the time over which each switch is closed, with a mean equal to $t_{\mathrm{ON}}=42.5 \mathrm{ps}\left(t_{\mathrm{ON}}=T_{s} / N-20 \mathrm{ps}\right.$ with $N=16$ paths $)$ and a standard deviation of 3 ps. For comparison, along with realistic scattering parameters plotted in color, we also plot their idealized counterparts as light gray shadow curves [see Figs. 1(e) and 8(b), and $9(\mathrm{~b})]$.

signal can also affect the SNR of the input signal. Nevertheless, past implementations of commutated networks $[42,43]$ show that clocking circuits can be realized with very small phase noise such that it has very little impact on the overall SNR of the input signal.

To quantitatively assess the influence of nonidealities, in Fig. 10, we compute the scattering parameters of the commutate network, the rat-race coupler, and the circulator discussed earlier, for the case of a nonzero on switch resistance, finite rise and fall times, and in the presence of clocking noise which affects the time over which each switch is closed. It is seen that for all devices the insertion loss is increased by only about $1-1.5 \mathrm{~dB}$. For the rat-race coupler and circulator, the reduction of isolation is the most noticeable effect, but for both devices more than $20 \mathrm{~dB}$ isolation is still achieved. It turns out, however, that this isolation reduction can be partially recouped by retuning the device parameters such as the capacitance, inductance, and commutation frequency. Most importantly, it is seen that the presence of nonidealities does not affect the transmission properties of all devices, meaning that the linear phase profile of commutated networks remains intact.

\section{CONCLUSIONS AND OUTLOOK}

In this work, we introduced the concept of quasielectrostatic wave propagation in time-modulated circuits that support extremely broadband slow-wave propagation overcoming the delay-bandwidth product bounds of linear time-invariant devices. Our findings leverage the existing framework of $N$-path filters in integrated electronics and address important needs in the field of communications and computing in overcoming the delay-bandwidth product of time-invariant systems. The commutated multipath networks implementing this concept provide reconfigurable reciprocal and nonreciprocal phase shifts and group delays that depend solely on the modulation frequency and commutation phase shift. We have shown that such networks exhibit nearly uniform ultrawideband transmission with near-zero group velocity dispersion. Using these findings, we demonstrated novel topologies for fully integrable reciprocal and nonreciprocal devices, largely reconfigurable in real time. The fact that commutated multipath networks can provide large reciprocal and nonreciprocal phase shifts without the use of large inductors offers unprecedented opportunities for miniaturization and on-chip integration of a wide range of electronic devices.

Finally, this work shows that temporal modulation not only allows overcoming the delay-bandwidth product of time-invariant systems, but also reveals that the achievable DBP in time-modulated systems does not have a fundamental upper bound. In view of this property, even though a direct translation of the approach presented in this work to optical systems operating at hundreds of terahertz is not straightforward, we envision that our work may inspire interesting developments in slow-light photonic systems employing novel spatiotemporal modulation schemes and multipath topologies.

\section{ACKNOWLEDGMENTS}

This work was supported by the DARPA SPAR program and the Air Force Office of Scientific Research with MURI Grant No. FA9550-18-1-0379.

\section{APPENDIX: LTP SYSTEMS ANALYSIS USING FLOQUET SCATTERING MATRICES}

Theoretical and numerical analyses of a complex linear time-periodic (LTP) electronic and photonic system is nontrivial. Even for relatively simple systems such as electronic circuits, the complexity of the problem grows dramatically with the increase of the number of circuit nodes and frequency harmonics included in the analysis. 
Additionally, while there are several commercial packages able to accurately treat LTP networks, none of them offer a straightforward and rigorous way to analyze the properties of an infinite cascade of LTP unit cells. Here, we show a rigorous divide-and-conquer method employing Floquet scattering matrices for the analysis of standalone and cascaded LTP networks which can be applied to both electronic and photonic systems. The main advantage of this method is the possibility to analyze each subnetwork independently and then gradually assemble the final circuit connecting only few elements at a time, that allowed us to keep the computational cost low.

Consider a two-port LTI network, the response of which can be fully characterized through its scattering matrix $S_{i j}$ in the frequency domain:

$$
\left(\begin{array}{l}
a_{1}^{-} \\
a_{2}^{-}
\end{array}\right)=\left[\begin{array}{ll}
S_{11} & S_{12} \\
S_{21} & S_{22}
\end{array}\right]\left(\begin{array}{c}
a_{1}^{+} \\
a_{2}^{+}
\end{array}\right),
$$

where $a_{i}^{+}$and $a_{i}^{-}$are power wave phasors for incoming and outgoing signals from the $i$ th port [58], respectively. If the system is modulated in time with an angular frequency $\omega_{s}=2 \pi / T_{s}$, these signals become time dependent, and they can be presented as a superposition of Floquet states (sometimes called tones) with frequencies $\omega+n \omega_{s}$ :

$$
a_{i}^{ \pm}(t)=\sum_{n=-\infty}^{\infty} a_{i, n}^{ \pm} e^{j\left(\omega+n \omega_{s}\right) t},
$$

where $a_{i, n}$ are the corresponding Fourier amplitudes. Similarly to the static case, we can follow the definition of power waves [58] and introduce generalized Floquet scattering parameters $S_{i j, m n}=a_{i, m}^{-} / a_{j, n}^{+}$, where $i, j$ denote the port numbers and $m, n$ are the corresponding harmonics. For simplicity, we assume that input and output ports have the same real impedance $Z_{0}$. Arranging the coefficients into vectors, $\mathbf{a}_{i}^{ \pm}=\left(\ldots, a_{i,-2}^{ \pm}, a_{i,-1}^{ \pm}, a_{i, 0}^{ \pm}, a_{i, 1}^{ \pm}, a_{i, 2}^{ \pm}, \ldots\right)^{T}$, the system (A1) can be presented in a block-matrix form:

$$
\left(\begin{array}{c}
\mathbf{a}_{1}^{-} \\
\mathbf{a}_{2}^{-}
\end{array}\right)=\left[\begin{array}{ll}
\mathbf{S}_{11} & \mathbf{S}_{12} \\
\mathbf{S}_{21} & \mathbf{S}_{22}
\end{array}\right]\left(\begin{array}{c}
\mathbf{a}_{1}^{+} \\
\mathbf{a}_{2}^{+}
\end{array}\right),
$$

where, for example, $\mathbf{S}_{11}$ is a square Floquet scattering matrix (FSM) containing reflection coefficients from the $n$th to the $m$ th harmonic at port $1, S_{11, m n}$. For simple elements such as inductors, capacitors, resistances, etc., the corresponding FSMs can be evaluated analytically through their conversion matrices $H_{i j, m n}$ [59], which are related to Floquet scattering parameters as

$$
S_{i j, m n}=2 H_{i j, m n}-\delta_{i j} \delta_{m n},
$$

where $\delta_{i j}$ is a Dirac delta function. As an example, we show the evaluation of the transfer function of a periodically

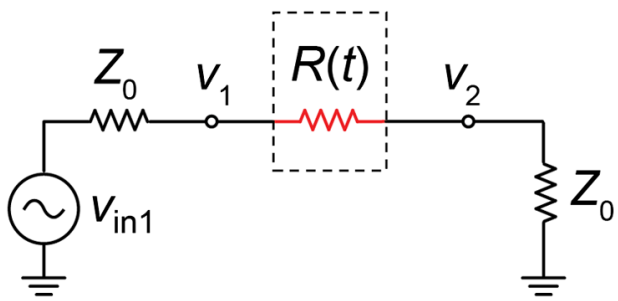

FIG. 11. Schematic of a time-varying resistance used to model a switch, connected to a voltage source through the impedance $Z_{0}$. The load impedance is also $Z_{0}$.

modulated resistance which we used in this paper to model a switch.

Consider a network depicted in Fig. 11, with a voltage source connected to port 1. Applying KCL in the time domain, we find

$$
\begin{aligned}
\frac{v_{\text {in }}-v_{1}}{Z_{0}} & =\frac{v_{1}-v_{2}}{R}=\frac{v_{2}}{Z_{0}}, \\
v_{\text {in }} & =v_{1}+v_{2} .
\end{aligned}
$$

Combining these two equations, we derive

$$
\begin{aligned}
& v_{2}=\frac{Z_{0}}{2 Z_{0}+R(t)} v_{\text {in }}=h_{21} v_{\text {in }}, \\
& v_{1}=\frac{Z_{0}+R(t)}{2 Z_{0}+R(t)} v_{\text {in }}=h_{11} v_{\text {in }} .
\end{aligned}
$$

We may now expand the time-dependent transfer functions into a Fourier series:

$$
h_{i j}(t)=\sum_{m=-\infty}^{\infty} H_{i j, m} e^{j m \omega_{s} t},
$$

with

$$
H_{i j, m}=\frac{1}{T_{s}} \int_{-T_{s} / 2}^{T_{s} / 2} h_{i j}(t) e^{-j m \omega_{s} t} d t .
$$

Substituting Eqs. (A2) and (A9) into Eqs. (A7) and (A8) and invoking the Cauchy convolution rule, we obtain

$$
\begin{aligned}
& V_{2, m}=H_{21, m n} V_{\mathrm{in} 1, n}, \\
& V_{1, m}=H_{11, m n} V_{\mathrm{in} 1, n},
\end{aligned}
$$

where $H_{i j, m n} \equiv H_{i j, m-n}$ is a Toeplitz matrix composed of Fourier coefficients. Because of network symmetry, $H_{12, m n}=H_{21, m n}$ and $H_{22, m n}=H_{11, m n}$, and, having all elements of $H_{i j, m n}$, the Floquet scattering matrix can be easily evaluated through Eq. (A4). Representation (A3) fully describes the time-varying behavior of the network at all frequencies. It also becomes evident that any LTP system can be treated as a time-invariant network with an 
infinite number of virtual frequency ports per each physical port. Similarly, FSMs can be derived for time-modulated systems having more than two physical ports.

It is now possible to derive a composite FSM of a complex network using, for example, a standard starproduct cascading procedure [60], given that the FSMs of the system's components are calculated in the same frequency basis. Instead of cascading, however, here we use another method enabling us to connect multiple FSMs simultaneously. Aggregating FSMs relating all incoming and outgoing waves existing within the system, we can generally write

$$
\mathbf{a}_{i}^{-}=\sum_{j} \mathbf{S}_{i j} \mathbf{a}_{j}^{+}
$$

The port indexes in Eq. (A13) can be split into two subsets corresponding to inner ports further denoted with small letters $p, q$ and outer ports denoted with capital letters $P, Q$. Accordingly, we can split Eq. (A13) as

$$
\begin{aligned}
& \mathbf{a}_{p}^{-}=\sum_{q} \mathbf{S}_{p q} \mathbf{a}_{q}^{+}+\sum_{Q} \mathbf{S}_{p Q} \mathbf{a}_{Q}^{+}, \\
& \mathbf{a}_{P}^{-}=\sum_{q} \mathbf{S}_{P q} \mathbf{a}_{q}^{+}+\sum_{Q} \mathbf{S}_{P Q} \mathbf{a}_{Q}^{+} .
\end{aligned}
$$

The fact that the inner ports are interconnected gives us another equation:

$$
\mathbf{a}_{p}^{-}=\sum_{q} \mathbf{F}_{p q} \mathbf{a}_{q}^{+}
$$

where $\mathbf{F}$ is a highly sparse matrix defining the relations among the modes at inner ports and nodes. For example, if port 2 is connected to an impedance-matched port 3 , we should enforce $\mathbf{a}_{2}^{-}=\mathbf{a}_{3}^{+}$and $\mathbf{a}_{3}^{-}=\mathbf{a}_{2}^{+}$, leading to $\mathbf{F}_{23}=\mathbf{F}_{32}=\mathbf{I}$, where $\mathbf{I}$ is a unitary matrix. Combining the system (A14) and Eq. (A15), we can find a composite FSM, $\tilde{\mathbf{S}}_{P Q}$, relating only the outer ports, $\mathbf{a}_{P}^{-}=\sum_{Q} \tilde{\mathbf{S}}_{P Q} \mathbf{a}_{Q}^{+}$:

$$
\tilde{\mathbf{S}}_{P Q}=\mathbf{S}_{P Q}+\sum_{q} \mathbf{S}_{P q} \sum_{p}\left[\mathbf{F}_{p q}-\mathbf{S}_{p q}\right]_{q p}^{-1} \mathbf{S}_{p Q}
$$

where $[.]^{-1}$ denotes an inverted matrix. Throughout this work, we use Eq. (A16) with 64 harmonics ( $m, n \in$ $[-64, \ldots, 0, \ldots, 64])$ for the analysis of all LTP circuits.

Now, we can proceed to analyzing the properties of a cascaded network of LTP subnetworks, each of which is characterized by its composite FSM (A16). The conventional method of analysis of periodic networks through their transfer matrixes [53] is not applicable in this case, because it involves an inverse of $\mathbf{S}_{21}$ block matrix, which is not guaranteed to exist. Instead, let us rearrange Eq. (A3) as

$$
\left[\begin{array}{ll}
-\mathbf{I} & \mathbf{S}_{11} \\
& \mathbf{S}_{21}
\end{array}\right]\left(\begin{array}{c}
\mathbf{a}_{1}^{-} \\
\mathbf{a}_{1}^{+}
\end{array}\right)=\left[\begin{array}{ll}
-\mathbf{S}_{12} & \\
-\mathbf{S}_{22} & \mathbf{I}
\end{array}\right]\left(\begin{array}{c}
\mathbf{a}_{2}^{+} \\
\mathbf{a}_{2}^{-}
\end{array}\right)
$$

Applying the Bloch theorem, we can also write

$$
\left(\begin{array}{l}
\mathbf{a}_{2}^{+} \\
\mathbf{a}_{2}^{-}
\end{array}\right)=e^{j \psi_{B}}\left(\begin{array}{c}
\mathbf{a}_{1}^{-} \\
\mathbf{a}_{1}^{+}
\end{array}\right)
$$

where $\psi_{B}$ is a Bloch phase accumulated after propagation over each unit cell. Substituting Eq. (A18) into (A17), we can pose an eigenvalue problem:

$$
\left[\begin{array}{ll}
-\mathbf{S}_{12} & \\
-\mathbf{S}_{22} & \mathbf{I}
\end{array}\right]^{-1}\left[\begin{array}{ll}
-\mathbf{I} & \mathbf{S}_{11} \\
& \mathbf{S}_{21}
\end{array}\right]\left(\begin{array}{c}
\mathbf{a}_{1}^{-} \\
\mathbf{a}_{1}^{+}
\end{array}\right)=e^{j \psi_{B}}\left(\begin{array}{c}
\mathbf{a}_{1}^{-} \\
\mathbf{a}_{1}^{+}
\end{array}\right),
$$

which yields a complete set of all possible Bloch phase shifts, time-varying power wave amplitudes, and Bloch impedances of the network.

[1] K. L. Tsakmakidis, O. Hess, R. W. Boyd, and X. Zhang, Ultraslow Waves on the Nanoscale, Science 358, eaan5196 (2017).

[2] T. Baba, Slow Light in Photonic Crystals, Nat. Photonics 2, 465 (2008).

[3] T. F. Krauss, Why Do We Need Slow Light?, Nat. Photonics 2, 448 (2008).

[4] L. Thévenaz, Slow and Fast Light in Optical Fibres, Nat. Photonics 2, 474 (2008).

[5] R. W. Boyd, Slow and Fast Light: Fundamentals and Applications, J. Mod. Opt. 56, 1908 (2009).

[6] K. L. Tsakmakidis, A.D. Boardman, and O. Hess, "Trapped Rainbow" Storage of Light in Metamaterials, Nature (London) 450, 397 (2007).

[7] Q. Gan, Z. Fu, Y. J. Ding, and F. J. Bartoli, UltrawideBandwidth Slow-Light System Based on THz Plasmonic Graded Metallic Grating Structures, Phys. Rev. Lett. 100, 256803 (2008).

[8] H. Hu, D. Ji, X. Zeng, K. Liu, and Q. Gan, Rainbow Trapping in Hyperbolic Metamaterial Waveguide, Sci. Rep. 3, 1249 (2013).

[9] M. I. Stockman, Nanofocusing of Optical Energy in Tapered Plasmonic Waveguides, Phys. Rev. Lett. 93, 137404 (2004).

[10] K. L. Tsakmakidis, L. Shen, S. A. Schulz, X. Zheng, J. Upham, X. Deng, H. Altug, A. F. Vakakis, and R. W. Boyd, Breaking Lorentz Reciprocity to Overcome the TimeBandwidth Limit in Physics and Engineering, Science 356, 1260 (2017).

[11] S. A. Mann, D. L. Sounas, and A. Alù, Nonreciprocal Cavities and the Time-Bandwidth Limit, Optica 6, 104 (2019).

[12] M. F. Yanik and S. Fan, Stopping and Storing Light Coherently, Phys. Rev. A 71, 013803 (2005).

[13] D. A. B. Miller, Fundamental Limit to Linear OneDimensional Slow Light Structures, Phys. Rev. Lett. 99, 203903 (2007). 
[14] C. Thakkar, N. Narevsky, C. D. Hull, and E. Alon, Design Techniques for a Mixed Signal I/Q 32-Coefficient RxFeedforward Equalizer, 100-Coefficient Decision Feedback Equalizer in an $8 \mathrm{~Gb} / \mathrm{s} 60 \mathrm{GHz} 65 \mathrm{~nm}$ LP CMOS Receiver, IEEE J. Solid-State Circuits 49, 2588 (2014).

[15] J. Roderick, H. Krishnaswamy, K. Newton, and H. Hashemi, Silicon-based Ultra-Wideband Beam-Forming, IEEE J. Solid-State Circuits 41, 1726 (2006).

[16] E. Everett, A. Sahai, and A. Sabharwal, Passive SelfInterference Suppression for Full-Duplex Infrastructure Nodes, IEEE Trans. Wireless Commun. 13, 680 (2014).

[17] Y. Okawachi, M. S. Bigelow, J. E. Sharping, Z. Zhu, A. Schweinsberg, D. J. Gauthier, R. W. Boyd, and A. L. Gaeta, Tunable All-Optical Delays via Brillouin Slow Light in an Optical Fiber, Phys. Rev. Lett. 94, 153902 (2005).

[18] K. Y. Song, M. G. Herráez, and L. Thévenaz, Gain-Assisted Pulse Advancement Using Single and Double Brillouin Gain Peaks in Optical Fibers, Opt. Express 13, 9758 (2005).

[19] K. Y. Song, K. S. Abedin, K. Hotate, M. G. Herráez, and L. Thévenaz, Highly Efficient Brillouin Slow and Fast Light Using $\mathrm{As}_{2} \mathrm{Se}_{3}$ Chalcogenide Fiber, Opt. Express 14, 5860 (2006).

[20] D. Dahan and G. Eisenstein, Tunable All Optical Delay via Slow and Fast Light Propagation in a Raman Assisted Fiber Optical Parametric Amplifier: A Route to All Optical Buffering, Opt. Express 13, 6234 (2005).

[21] J. E. Sharping, Y. Okawachi, and A. L. Gaeta, Wide Bandwidth Slow Light Using a Raman Fiber Amplifier, Opt. Express 13, 6092 (2005).

[22] M. F. Yanik and S. Fan, Stopping Light All Optically, Phys. Rev. Lett. 92, 083901 (2004).

[23] F. Masetti and P. Gavignet-Morin, Optical Fiber Buffer for High-Performance Broadband Switching, European Transactions on Telecommunications and Related Technologies 4, 671 (1993).

[24] M. Tsukada, A. Misawa, J. Nishikido, Y. Shimazu, and H. Nakano, Experiments on Photonic Cell Switching with an Optical Input Buffer, Electron. Lett. 30, 1081 (1994).

[25] M. Calzavara, P. Gambini, M. Puleo, M. Burzio, P. Cinato, E. Vezzoni, F. Delorme, and H. Nakajima, Resolution of ATM Cell Contention by Multiwavelength Fiber loop memory, in Proc. Photon. Switching/Eur. Conf. Opt. Commun., ECOC'94 2, 567 (1994).

[26] R. Langenhorst, M. Eiselt, W. Pieper, G. Großkopf, R. Ludwig, L. Küller, E. Dietrich, and H. G. Weber, Fiber Loop Optical Buffer, J. Lightwave Technol. 14, 324 (1996).

[27] D. Grassani, I. Cardea, S. J. Fabbri, J. Upham, R. W. Boyd, H. Altug, S. A. Schulz, K. L. Tsakmakidis, and C.-S. Brès, Demonstration of Ultra-high Time-Bandwidth Product in a Non-reciprocal Fiber-Optic System, in Proceedings of Frontiers in Optics/Laser Science (Optical Society of America, Washington, DC, 2018), paper JTu3A.32.

[28] Q. Xu, P. Dong, and M. Lipson, Breaking the DelayBandwidth Limit in a Photonic Structure, Nat. Phys. 3, 406 (2007).

[29] Y. Tanaka, J. Upham, T. Nagashima, T. Sugiya, T. Asano, and S. Noda, Dynamic Control of the $Q$ Factor in a Photonic Crystal Nanocavity, Nat. Mater. 6, 862 (2007).
[30] Some nonresonant light-trapping systems, such as wedge states fed by nonreciprocal unidirectional waveguides, can also release the trapped pulse by dynamically reversing the direction of unidirectional mode propagation [10]. This fact has led to some confusion on whether the DBP limit may be broken in time-invariant nonreciprocal systems. However, as explained in Ref. [11], reciprocity breaking alone does not provide specific advantages to increase the DBP. It is temporal variations that enable enhancing the DBP in these systems.

[31] Z. Yu and S. Fan, Complete Optical Isolation Created by Indirect Interband Photonic Transitions, Nat. Photonics 3, 91 (2009).

[32] Z. Yu and S. Fan, Integrated Nonmagnetic Optical Isolators Based on Photonic Transitions, IEEE J. Sel. Top. Quantum Electron. 16, 459 (2010).

[33] K. Fang, Z. Yu, and S. Fan, Photonic Aharonov-Bohm Effect Based on Dynamic Modulation, Phys. Rev. Lett. 108, 153901 (2012).

[34] D. L. Sounas and A. Alù, Angular-Momentum-Biased Nanorings to Realize Magnetic-Free Integrated Optical Isolation, ACS Photonics 1, 198 (2014).

[35] L. D. Tzuang, K. Fang, P. Nussenzveig, S. Fan, and M. Lipson, Nonreciprocal Phase Shift Induced by an Effective Magnetic Flux for Light, Nat. Photonics 8, 701 (2014).

[36] N. A. Estep, D. L. Sounas, J. Soric, and A. Alù, MagneticFree Nonreciprocity and Isolation Based on Parametrically Modulated Coupled-Resonator Loops, Nat. Phys. 10, 923 (2014).

[37] N. A. Estep, D. L. Sounas, and A. Alu, Magnetless Microwave Circulators Based on Spatiotemporally Modulated Rings of Coupled Resonators, IEEE Trans. Microwave Theory Tech. 64, 1 (2016).

[38] A. Kord, D. Sounas, and A. Alu, Achieving Full-Duplex Communication: Magnetless Parametric Circulators for Full-Duplex Communication Systems, IEEE Microw. Mag. 19, 84 (2018).

[39] A. Kord, D. Sounas, and A. Alu, Magnetless Circulators Based on Spatio-Temporal Modulation of Bandstop Filters in a Delta Topology, IEEE Trans. Microwave Theory Tech. 66, 911 (2018).

[40] N. Reiskarimian and H. Krishnaswamy, Magnetic-Free Nonreciprocity Based on Staggered Commutation, Nat. Commun. 7, 11217 (2016).

[41] T. Dinc, M. Tymchenko, A. Nagulu, D. Sounas, A. Alu, and H. Krishnaswamy, Synchronized Conductivity Modulation to Realize Broadband Lossless Magnetic-Free Nonreciprocity, Nat. Commun. 8, 795 (2017).

[42] T. Dinc, A. Nagulu, and H. Krishnaswamy, A MillimeterWave Non-Magnetic Passive SOI CMOS Circulator Based on Spatio-Temporal Conductivity Modulation, IEEE J. Solid-State Circuits 52, 3276 (2017).

[43] A. Nagulu, T. Dinc, Z. Xiao, M. Tymchenko, D. Sounas, A. Alu, and H. Krishnaswamy, Nonreciprocal Components Based on Switched Transmission Lines, IEEE Trans. Microwave Theory Tech. 66, 4706 (2018).

[44] M. M. Biedka, R. Zhu, Q. M. Xu, and Y. E. Wang, UltraWide Band Nonreciprocity through Sequentially-Switched Delay Lines, Sci. Rep. 7, 40014 (2017).

[45] N. Reiskarimian, J. Zhou, T.-H. Chuang, and H. Krishnaswamy, Analysis and Design of Two-Port N-Path 
Bandpass Filters with Embedded Phase Shifting, IEEE Trans. Circuits Syst. II 63, 728 (2016).

[46] A. Ghaffari, E. A. M. Klumperink, M. C. M. Soer, and B. Nauta, Tunable High-Q N-Path Band-Pass Filters: Modeling and Verification, IEEE J. Solid-State Circuits 46, 998 (2011).

[47] Cadence, Spectre Circuit Simulator 6.10, https://www .cadence.com.

[48] L. E. Franks and I. Sandberg, An Alternative Approach to the Realization of Network Transfer Functions: The N-Path Filter, Bell Syst. Tech. J. 39, 1321 (1960).

[49] Note that we deliberately omit another reciprocal scenario, achieved for $\Delta T=0$, in which the two switches on the same branch close simultaneously. In this regime, the response of the network is drastically different and goes beyond the scope of this paper.

[50] M. Pelgrom, Analog-to-Digital Conversion, 3rd ed. (Springer, New York, 2017).

[51] Keysight Technologies, Advanced Design System (ADS) 2014, https://www.keysight.com.
[52] C. E. Shannon, Communication in the Presence of Noise, Proc. IRE 37, 10 (1949).

[53] D. Pozar, Microwave Engineering, 4th ed. (Wiley, New York, 2011).

[54] H. B. Bakoglu, Circuits, Interconnections, and Packaging for VLSI (Addison-Wesley, Reading, MA, 1990).

[55] B. R. Vasant, Delay Analysis of the Distributed RC Line, in Proceedings of the 32nd Design Automation Conference, San Francisco, CA, USA (IEEE, 1995), pp. 370-375.

[56] A. Kord, D. L. Sounas, and A. Alù, IEEE Trans. Microwave Theory Tech. 66, 2731 (2018).

[57] F. Yuan, CMOS Active Inductors and Transformers, 1st ed. (Springer, New York, 2008).

[58] K. Kurokawa, Power Waves and the Scattering Matrix, IEEE Trans. Microwave Theory Tech. 13, 194 (1965).

[59] S. A. Maas, Nonlinear Microwave, and RF Circuits, 2nd ed. (Artech House, Norwood, MA, 2003).

[60] P. L. Overfelt and D. J. White, Alternate Forms of the Generalized Composite Scattering Matrix, IEEE Trans. Microwave Theory Tech. 37, 1267 (1989). 